\title{
Mecanismos de resistencia a fluconazol expresados por Candida glabrata: una situación para considerar en la terapéutica*
}

Resistance mechanisms to fluconazole expressed by Candida glabrata: a situation to consider in therapy Mecanismos de resistência a fluconazol expressos por Candida glabrata: uma situação a considerar na terapêutica

\author{
Leidy Yurani Cárdenas Parra ${ }^{\text {a }}$ \\ Universidad de Caldas y Universidad Católica de \\ Manizales, Colombia \\ leidy.cardenas@ucaldas.edu.co \\ ORCID: https://orcid.org/0000-0001-7505-8539
}

DOI: https://doi.org/10.11144/Javeriana.ie22.mrfe

\author{
Jorge Enrique Pérez Cárdenas \\ Universidad de Caldas, Colombia \\ ORCID: https://orcid.org/0000-0002-7829-6505
}

\author{
Recibido: 10 Septiembre 2019 \\ Aceptado: 11 Mayo 2020 \\ Publicado: 22 Julio 2020
}

\section{Resumen:}

Introducción: Los esfuerzos terapéuticos orientados a atender las micosis por Candidaspp. se han enfocado en el empleo de azoles; sin embargo, en la literatura científica se discute su beneficio, por los amplios y descritos mecanismos de resistencia. Objetivo: Describir los mecanismos de resistencia al fluconazol expresados por la especie Candida glabrata, con la intención de que sean considerados dentro de las variables de elegibilidad para la intervención. Método: Se realizó una revisión integrativa utilizando la pregunta orientadora: ¿cuáles son los mecanismos de resistencia al fluconazol expresados por la especie Candida glabrata? Veintinueve estudios obtenidos de la base de datos PubMed cumplieron los criterios del análisis crítico propuesto por el instrumento PRISMA, utilizado para la selección de los artículos incluidos para su revisión en este manuscrito. Las categorías bajo las cuales se organizaron los elementos de análisis fueron: sobrexpresión de bombas de eflujo y modificaciones en la enzima lanosterol 14alfa-desmetilasa. Resultados: Los mecanismos de resistencia al fluconazol expresados por Candida glabrata están determinados principalmente por la regulación a la alza de bombas de adenosina-trifosfato Binding Cassette (ABC) y por la modificación del punto de unión con su blanco farmacológico: la enzima lanosterol 14-alfa-desmetilasa. Conclusión: Los mecanismos de resistencia expresados por Candida glabrata se asocian con la modificación estructural de la diana farmacológica y la sobreexpresión de bombas de eflujo de manera diferencial a otras especies. Se sugiere que Candida glabrata es intrínsecamente menos susceptible al fluconazol. Palabras clave: Candida glabrata, Candida, resistencia, azoles, fluconazol.

\section{Abstract:}

Introduction: Therapeutic efforts aimed at treating mycosis caused by Candida spp. have focused on the use of azoles; however, their benefits have been subject to discussion in scientific literature, due to the extensive and well-described resistance mechanisms. Objective: To describe the resistance mechanisms to fluconazole expressed by the Candida glabrata species, so they are considered within the variables of eligibility for intervention. Method: An integrative review was carried out using the guiding question: what are the fluconazole resistance mechanisms expressed by the Candida glabrata species? Twenty-nine studies obtained from the PubMed database met the criteria for the critical analysis proposed by the PRISMA instrument, which was used for the selection of articles for review included in this paper. The analysis elements were organized in the following categories: overexpression of efflux pumps and modifications in the enzyme lanosterol 14-alpha-demethylase. Results: The resistance mechanisms to fluconazole expressed by Candida glabrata are mainly determined by the upregulation of Adenosine triphosphate Binding Cassette (ABC) pumps and by the modification of the point of attachment with its pharmacological target: the enzyme lanosterol 14-alphademethylase. Conclusion: The resistance mechanisms expressed by Candida glabrata are associated with the structural modification of the pharmacological target and the overexpression of efflux pumps, in a way different to other species. It is suggested that Candida glabrata is intrinsically less susceptible to fluconazole.

Keywords: Candida glabrata, Candida; resistance; azoles; fluconazole.

Notas de autor

a Autora de correspondencia. Correo electrónico: leidy.cardenas@ucaldas.edu.co 


\section{Resumo:}

Introdução: Os esforços terapêuticos voltados ao tratamento de micose por Candida spp. se focaram no uso de azóis; no entanto, na literatura científica discute-se seu benefício devido aos extensos e descritos mecanismos de resistência. Objetivo: Descrever os mecanismos de resistência ao fluconazol expressos pela espécie Candida glabrata, com a intenção de serem considerados dentro das variáveis de elegibilidade para a intervenção. Método: Uma revisão integrativa foi realizada utilizando a questão norteadora: quais os mecanismos de resistência ao fluconazol expressos pela espécie Candida glabrata? Vinte e nove estudos obtidos da base de dados PubMed atenderam os critérios de análise crítica proposta pelo instrumento PRISMA, utilizado para a seleção dos artigos incluídos para revisão neste manuscrito. As categorias sob as quais se organizaram os elementos de análise foram: superexpressão de bombas de efluxo e modificações na enzima lanosterol 14-alfa-desmetilase. Resultados: Os mecanismos de resistência ao fluconazol expressos por Candida glabrata são determinados principalmente pela regulação positiva das bombas de adenosinatrifosfato Binding Cassette $(\mathrm{ABC})$ e pela modificação do ponto de fixação com seu alvo farmacológico: a enzima lanosterol 14alfa-desmetilasa. Conclusão: Os mecanismos de resistência expressos por Candida glabrata são associados à modificação estrutural da Diana farmacológica e a superexpressão de bombas de efluxo de maneira diferencial a outras espécies. Sugere-se que Candida glabrata é intrinsecamente menos susceptível ao fluconazol.

Palavras-chave: Candida glabrata, Candida, resistência, azóis, fluconazol.

\section{Introducción}

La epidemiología de las especies de Candida es variable en las diferentes regiones geográficas y grupos etarios. Se asocia con la exposición de personas susceptibles por su estado de salud a ciertos ambientes favorables para la aparición de este tipo de infecciones y se informa que este agente etiológico ha generado una amplia resistencia hacia algunos fármacos, lo cual dificulta la intervención terapéutica. Con relación a lo anterior y al revisar la literatura, se identificó que se ha estudiado con mayor frecuencia la resistencia de este microrganismo al fluconazol en Estados Unidos, seguido de Reino Unido, España, Suiza y Taiwán.

Los hospitales son un importante reservorio de microrganismos oportunistas, y particularmente Candida spp. tiene una representación importante, como causante de infecciones asociadas a la atención en varios servicios intrahospitalarios. Algunas investigaciones han documentado que la especie más frecuentemente aislada es $C$. albicans, cuya mayor proporción se encuentra en el norte y centro de Europa y en Estados Unidos. En muchos estudios se identifica como segunda causa de candidemia a C. glabrata y otros a C. tropicalis. De estas dos especies, la primera se encuentra ampliamente distribuida en las mismas regiones que C. albicans y la segunda en Suramérica y Asia (1-3). Otras especies aisladas, a menudo, corresponden a $C$. parapsilosis en Suramérica, sur de Europa y varios lugares de Asia, y C. krusei, cuya frecuencia de aislamiento es relativamente baja en todas las regiones (1-9).

De todas las especies mencionadas, $C$. glabrata es considerada generalmente una especie de baja virulencia, pero que causa una mortalidad mayor que $C$. albicans; además, las infecciones por este agente son difíciles de tratar porque sus características genéticas le confieren por sí mismas resistencia a fármacos azólicos, principalmente al fluconazol $(3,10)$.

Un estudio realizado en Colombia documentó una elevada frecuencia de infecciones por $C$. albicans y un aislamiento de $C$. glabrata en el $9,5 \%$ de los casos en varias unidades de cuidado intensivo de la ciudad de Medellín. De ahí la necesidad de atender esta problemática, pues aunque se dispone de pocos estudios al respecto, es un agente infeccioso que se encuentra presente en nuestro país (6).

El fluconazol es uno de los medicamentos más utilizados para el abordaje terapéutico de la candidemia. Algunos autores reportan una prescripción que supera a la mitad del total de las formulaciones de antifúngicos, de manera que frente a este particular contexto sobre el empleo del fluconazol y la gran variedad de estudios que acusan una amplia resistencia farmacológica desarrollada por el patógeno, se requieren análisis y documentación al respecto que fundamente los criterios de elegibilidad farmacológica, en el marco de la intervención terapéutica frente a este tipo de infecciones (11).

En la literatura sobre el tema, Cándida glabrata se ha descrito como una levadura intrínsecamente menos susceptible al fluconazol (12), hallazgo que motiva un especial interés de documentar el tipo de 
alteraciones moleculares que le confieren resistencia a esta especie frente al medicamento enunciado y, de esta manera, aportar elementos a la comunidad científica que sean de utilidad para determinar el empleo de otras alternativas terapéuticas efectivas y que, como beneficio asociado a una adecuada elección farmacológica, disminuyan los sobrecostos al sistema por el uso de medicamentos ineficaces, así como los problemas relacionados con medicamentos, particularmente reacciones adversas medicamentosas tipo $\mathrm{F}$ (fallo terapéutico) $(13,14)$.

\section{Método}

Se llevó a cabo una revisión integrativa en la base de datos PubMed, cuya pregunta orientadora para la búsqueda fue: ¿cuáles son los mecanismos de resistencia al fluconazol expresados por la especie Candida glabrata? Los términos utilizados fueron Candida glabrata, resistencia, azoles, fluconazol (DeCS) // Candida glabrata, resistant, azoles, fluconazole (MeSH) en combinación con los operadores booleanos AND y OR en "todos los campos". Se aplicaron filtros para resultados en los últimos 10 años y en humanos, sin filtros de idioma. La operación de búsqueda fue ((Candida glabrata) AND (azoles resistant OR fluconazole resistant)) AND (fluconazol OR azoles))) en noviembre de 2019 y se repitió en marzo del 2020, con el fin de incluir otros estudios actualizados sobre el tema correspondiente.

Así, se obtuvieron 299 estudios que se vincularon al gestor bibliográfico Zotero (https://www.zotero.org/). Los artículos que incluyeron en el título o resumen mecanismos de resistencia al fluconazol expresados particularmente por la especie Candida glabratadirectamente pasaron a ser elegibles, y los que incluyeron mecanismos de resistencia a algún fármaco del grupo de los azoles por C. glabrata o cualquier tipo de Candida se incluyeron para su posterior revisión, y en caso de ser un tema afín y relevante se incluyeron en la lista de elegibles.

El análisis de la lectura crítica y la calidad de los estudios se dio acorde a los elementos planteados por los informes PRISMA (15). A partir de los hallazgos, se establecieron dos categorías de análisis: la primera de ellas corresponde a los elementos que generan resistencia por la sobrexpresión de bombas de eflujo, y la segunda, a los mecanismos que confieren resistencia por modificaciones en la enzima lanosterol 14-alfadesmetilasa (figura 1). Se excluyeron artículos que hacían referencia a mecanismos de resistencia al fluconazol o a otros azoles y con resistencia cruzada a este agente farmacológico, pero que no incluyeron las características particulares expresadas por la especie Candida glabrata. 


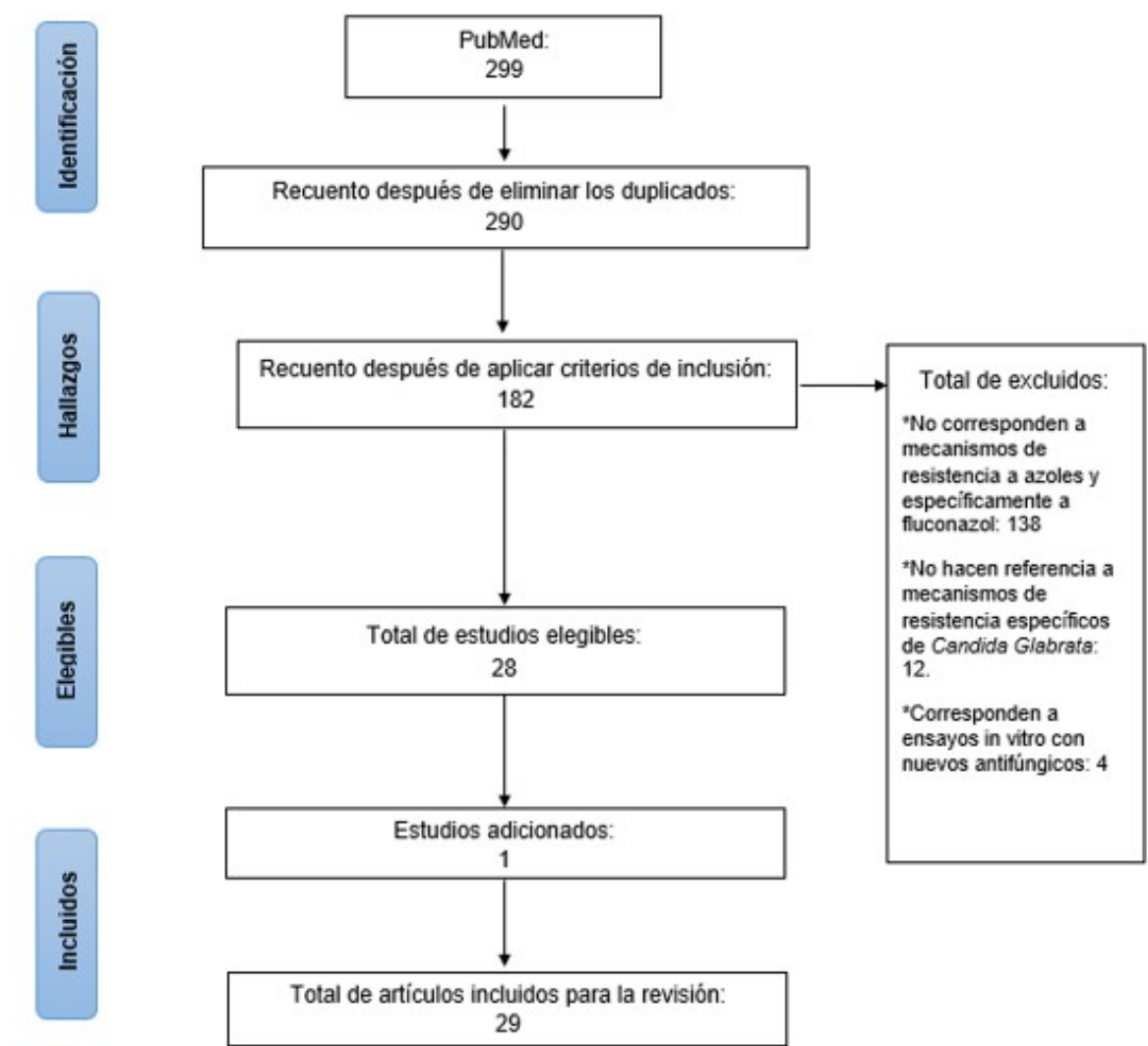

FIGURA 1.

Proceso de revisión de artículos-estudios basados en el método PRISMA (15) elementos de informes preferidos para revisiones sistemáticas y metanálisis: la declaración PRISMA (15)

\section{Resultados y discusión}

\section{Una revisión desde la perspectiva del agente etiológico: Candida glabrata}

Las levaduras del género Candidaforman parte de la microbiota normal de la piel, de la mucosa oral, del tejido gastrointestinal y vaginal del humano (16); pero por la interacción de diversos factores tanto de la levadura (virulencia y expresión de múltiples mecanismos de resistencia antifúngica) como del hospedero (hospitalización prolongada, enfermedad de base, inmunosupresión, amplia terapia antibimicrobiana, entre otros) se pueden producir infecciones sistémicas por este hongo (7).

Candida glabrata pertenece a la clase Deuteromycetes/fungi imperfecti, orden-forma Moniliales, familiaforma Cryptococcaceae.Su genoma está representado en un $40 \%$ por guanina-citosina y posee una homología en el gen varl del 85 \% con Saccharomyces cerevisiae,característica que le permite compartir con este hongo algunos mecanismos de resistencia farmacológica (17-19).

Es una especie de carácter haploide que tiende a sufrir más mutaciones con relación a especies diploides; es productora de colonias lisas de consistencia blanda y de color crema; presenta formas individuales ovoides, incapaces de formar seudohifas o seudomicelios o, como máximo, pueden formar una cadena corta de levaduras ovoides; su gemación es multilateral, no produce cápsula ni artrosporas y tampoco se han descrito esporas sexuales (7). 
Candida glabrata se aísla cada vez con mayor frecuencia en muestras clínicas. Está ampliamente asociada a micosis superficiales como candidiasis oral y vulvovaginal. Se ha descrito un incremento en el aislamiento de esta levadura en la sangre y en la orina, especialmente en pacientes inmunosuprimidos, diabéticos, con procesos neoplásicos y con exposición previa a antibioticoterapia múltiple de amplio espectro o al fluconazol. El uso de dispositivos médicos invasivos como catéter intravenoso o vesical aumenta el riesgo de adquirir dichas infecciones $(3,7)$.

El género Candida posee una pared celular compuesta fundamentalmente por polisacáridos, proteínas y lípidos que le confieren varias funciones, entre estas proteger al hongo de la pérdida osmótica, interactuar con el medio externo, favorecer el crecimiento celular, adherirse al hospedero, producir rigidez, tener propiedades enzimáticas y ayudar en la formación de biopelículas (20,21). A continuación, se describen los componentes más importantes de la pared y de la membrana celular de los hongos, por ser considerados dianas farmacológicas:

- Pared celular: el glucano es el principal polisacárido constitutivo de la pared y es el responsable de la rigidez de dicha estructura. En su síntesis intervienen las glucano-sintetasas que están reguladas por los genes FKS1, FKS2 y RHO (20,21). Un cambio en la expresión de estos podría modificar la farmacodinamia y volver resistente al hongo. Otro polisacárido es la manosa, la cual determina la porosidad de la pared y reconoce receptores en las células del hospedero y es fundamental en el proceso de adhesión $(22,23)$.

- Membrana celular: el ergosterol es el principal esterol de la bicapa lipídica de la membrana, esencial para brindarle integridad y funcionalidad; su síntesis está regulada por varios genes (ERG1 y ERG7, ERG24, ERG25, ERG26, ERG27, ERG6, ERG2, ERG3, ERG5, ERG4); sin embargo, el gen ERG11 desempeña un papel representativo en la codificación de lanosterol 14-alfa-desmetilasa, una enzima de la superfamilia del citocromo P450 (CYP) compuesta por 528 residuos de aminoácidos que se localiza en la membrana celular y tiene una función temprana en la biosíntesis de colesterol que consiste en desmetilar al lanosterol para formar ergosterol (7,24-26).

- Membrana celular: el ergosterol es el principal esterol de la bicapa lipídica de la membrana, esencial para brindarle integridad y funcionalidad; su síntesis está regulada por varios genes (ERG1. ERG7, ERG24, ERG25, ERG26, ERG27, ERG6, ERG2, ERG3, ERG5, ERG4); sin embargo, el gen ERG11 desempeña un papel representativo en la codificación de lanosterol 14-alfa-desmetilasa, una enzima de la superfamilia del citocromo P450 (CYP) compuesta por 528 residuos de aminoácidos que se localiza en la membrana celular y tiene una función temprana en la biosíntesis de colesterol que consiste en desmetilar al lanosterol para formar ergosterol (7,24-26).

Algunos aspectos específicos relacionados con la virulencia del hongo son la ausencia de seudohifas, lo que hace pensar que Candida glabrata es menos virulenta que otras especies; sin embargo, las pruebas de su frecuencia en candidemias sugiere una alta capacidad de diseminarse (7). Existen otros factores de virulencia como la capacidad de formar biopelículas o de modificar las adhesinas asociadas a la pared celular, su variabilidad morfológica, su adaptación al medio ácido de los fagolisosomas, la producción de pigmentos derivados del indol y su inherente tolerancia a azoles $(7,19,27-29)$.

\section{El fluconazol: primera línea de elección terapéutica frente a infecciones por Candida}

Los fármacos antimicóticos comúnmente usados para el tratamiento de infecciones fúngicas invasivas pertenecen a tres clases, caracterizadas por diferentes mecanismos de acción y espectro de actividad. Debido a la naturaleza eucariota común de las células fúngicas y humanas, es difícil identificar objetivos antimicrobianos metabólicos o estructurales específicos para los hongos. El papel fisiológico fundamental y la 
diferente composición de los esteroles (colesterol en los seres humanos y ergosterol en los hongos) hacen que la membrana citoplasmática de los hongos sea un objetivo adecuado para la acción de los antifúngicos (polienos y azoles) con un índice terapéutico suficiente. La síntesis de los glucanos de la pared celular representa un objetivo metabólico adicional, explorado por las equinocandinas (30).

Los azoles tienen un espectro antifúngico in vitro amplio que actúa sobre diferentes especies de hongos, entre ellos diversas especies de Candida (30). Este grupo farmacológico es considerado la primera línea de elección para el tratamiento de enfermedades micóticas y el fluconazol es el más frecuentemente utilizado (31). Dichos fármacos entran en la levadura por difusión facilitada y a través del nitrógeno de su estructura molecular se unen al grupo hemo de la lanosterol 14-alfa-desmetilasa y al interactuar con los residuos de aminoácidos de la estructura, disminuyen la afinidad de esta por el sustrato endógeno (32). Esta enzima es clave en la biosíntesis del ergosterol a partir de la desmetilación del lanosterol (33), pues al ser inhibida su actividad, se impide la formación de ergosterol y se acumulan esteroles 14-alfa-desmetilados a nivel intracelular, lo que ocasiona una afectación estructural de la membrana y, de manera adicional, genera varios fenómenos representados por el incremento de la permeabilidad del microrganismo (25). También hay una modificación del ambiente intracelular que altera el desarrollo y la división del hongo y la función de enzimas que participan en la cadena de transporte de electrones (30). Dichas modificaciones, tras la interacción con el antimicótico, anteceden la muerte del agente patógeno $(25,34)$.

Otros mecanismos de acción asociados a este grupo de medicamentos se relacionan con la acumulación de peróxido de hidrógeno secundario a la alteración de los mecanismos enzimáticos intracelulares que intervienen en su síntesis y desoxificación, lo que produce una acumulación neta capaz de lesionar las organelas intracelulares; sin embargo, como se mencionó con anterioridad a C. glabrata, se le atribuye una capacidad adaptativa a dicho ambiente ácido. El otro mecanismo corresponde a la inhibición de la enzima C-5 desaturasa, implicada en la deshidratación de un precursor del ergosterol, llamado episterol (34-36).

El mecanismo asociado a la enzima 14 alfa-desmetilasa es el más representativo en cuanto a la farmacodinamia del fluconazol. En su estructura, dicha enzima posee residuos de aminoácidos responsables de formar la superficie de unión de su sustrato, los cuales migran hasta el sitio activo donde ocurre la desmetilación en tres pasos en los cuales se requieren dos átomos de oxígeno y una molécula de NADPH. En los dos primeros pasos se incorpora un átomo de oxígeno al sustrato y el otro átomo de oxígeno se reduce en agua, lo que da como resultado un carboxialcohol y luego un carboxialdehído; este último en una etapa final, el grupo aldehído se convierte en ácido fórmico y se genera un doble enlace para crear el producto desmetilado (37).

\section{El fenómeno de la resistencia a los azoles, expresados por Candida glabrata}

La resistencia a los antifúngicos es una problemática global que dificulta la terapia farmacológica con los azoles y se debe a eventos multifactoriales que implican modificaciones moleculares y sobreexpresión de genes en el hongo, lo cual afecta la efectividad farmacodinámica del medicamento (32). La falla del tratamiento antifúngico puede estar relacionada con el hospedero (estado inmune, sitio de infección, gravedad de la infección, formación de abscesos, adherencia al régimen de tratamiento, actividad fungistática o fungicida, dosificación, farmacocinética, interacciones medicamentosas), pero también puede estar asociada con el hongo implicado (morfología, fenotipo, serotipo, estabilidad genómica, carga fúngica, producción de biopelículas) (38,39). En la última década, los roles fundamentales de los factores de transcripción y la plasticidad del genoma del agente patógeno se han destacado como los responsables de los procesos de resistencia antifúngica $(7,40-42)$.

En la mayoría de los estudios realizados por el Instituto de Estándares Clínicos y de Laboratorio, los valores de la concentración mínima inhibitoria (MIC) del fluconazol frente a C. glabrata estuvieron por encima del punto de ruptura susceptible $(\geq 16 \mu \mathrm{g} / \mathrm{ml})$; resultados que, desde el punto de vista terapéutico, 
llaman la atención, puesto que con relación a otras especies de Candidaspp., Candida glabrata muestra una marcada diferencia en cuanto a su respuesta ante este molécula terapéutica, lo que ha puesto de manifiesto que existen fenómenos de resistencia importantes y diferenciales relacionados con este agente infeccioso y que intrínsecamente es menos susceptible al fluconazol. En la tabla 1 se visualizan los criterios interpretativos de susceptibilidad para el fluconazol contra especies de Candida spp. según los estudios mencionados con anterioridad (43-46).

TABLA 1.

Criterios interpretativos de susceptibilidad para el fluconazol contra especies de Candida spp. (43-46)

\begin{tabular}{lrrrrrr}
\hline \multirow{2}{*}{ Especie de Candida a } & \multicolumn{2}{c}{$\begin{array}{c}\text { Microdilución en caldo a las 24 horas } \\
\text { (MIC en } \boldsymbol{\mu g} / \mathbf{m L} \text { ) }\end{array}$} & \multicolumn{3}{c}{$\begin{array}{c}\text { Difusión de disco a las 24 horas } \\
\text { (diámetros de la zona en mm) }\end{array}$} \\
\cline { 2 - 8 } & $\mathbf{S}$ & SDD & R & S & SDD & R \\
\hline C. albicans & $\leq 2$ & 4 & $\geq 8$ & $\geq 17$ & $14-16$ & $\leq 13$ \\
\hline C. glabrata & -- & $\leq 32$ & $\geq 64$ & - & $\geq 15$ & $\leq 14$ \\
\hline C. parapsilosis & $\leq 2$ & 4 & $\geq 8$ & $\geq 17$ & $14-16$ & $\leq 13$ \\
\hline C. tropicalis & $\leq 2$ & 4 & $\geq 8$ & $\geq 17$ & $14-16$ & $\leq 13$ \\
\hline
\end{tabular}

MIC: concentración mínima inhibitoria; S: susceptible; SDD: susceptible dependiente de dosis; R: resistente. Clinical and Laboratory Standards Institute

Candida glabrata posee mecanismos adquiridos de resistencia a azoles, representados por la inducción de bombas de eflujo codificadas por los genes $M D R$ o $C D R$ y mutaciones puntuales en el gen ERG11, que codifican para la diana farmacológica (lanosterol alfa-metiltransferasa) (47-51).

Las proteínas involucradas en el transporte y extrusión de azoles pertenecen a dos clases principales: una clase que utiliza el trifosfato de adenosina para realizar su función (las llamadas ABC, por sus siglas en inglés Adenosin Triphosphate Binding Cassette) y la clase denominada superfamilia mayor facilitadora (MFS de Major Facilitator Superfamily). Los fenómenos de resistencia en Candida glabrata se asocian con sobrexpresión de las bombas $\mathrm{ABC}$ que confieren resistencia cruzada a varios azoles, las cuales están reguladas en esta especie en particular por los genes $C g C D R 1, C g C D R 2, C g S N Q 2$, PDH1, CgPDR1, y en el caso de las bombas de salida MFS, los cambios se presentan en el gen $M D R 1$. La sobreexpresión de este tipo de transportadores limita el ingreso del fármaco al interior de la célula fúngica y este fenómeno se asocia con la resistencia del patógeno $(31,42,49,50,52-63)$ (tabla 2).

TABLA 2.

Síntesis de los mecanismos moleculares de resistencia expresados por Cándida glabrata (31,36,42,49-63)

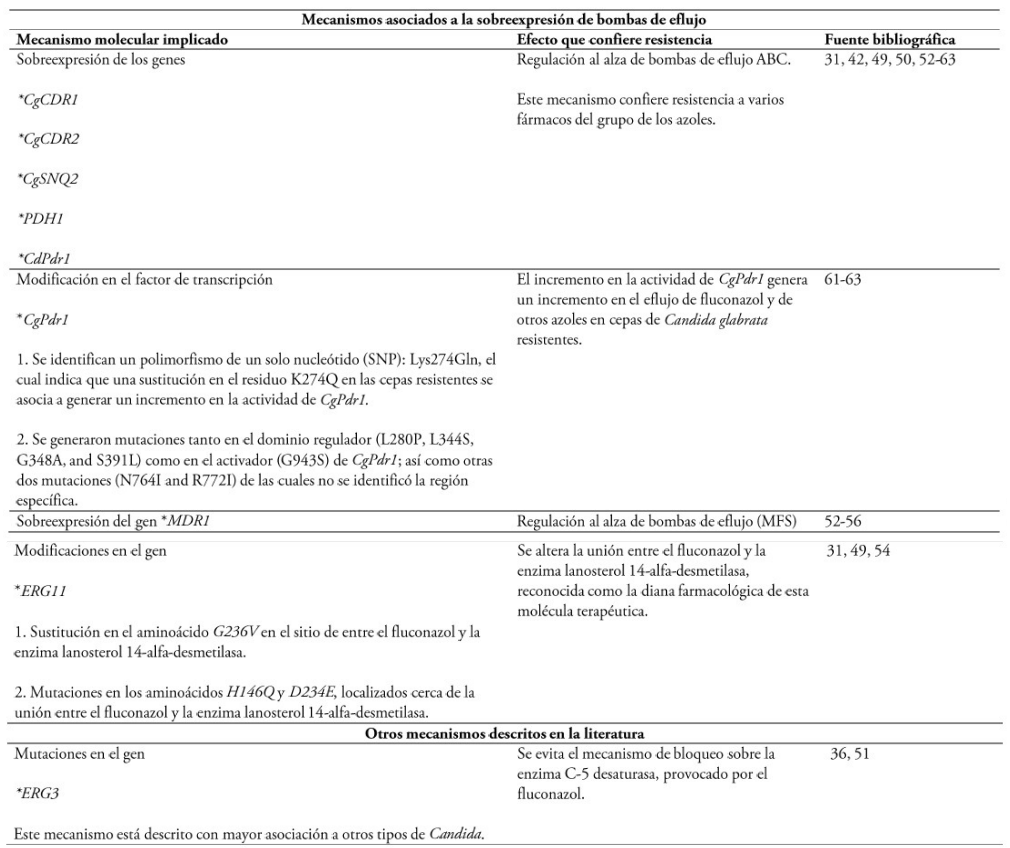

Fuente: elaboración propia 
Las mutaciones del gen $E R G 11$ se asocian a la disminución de la afinidad del fármaco por su diana biológica (enzima 14-alfa-desmetilasa), siendo este el mecanismo asociado a la resistencia antifúngica (49,54). En un estudio realizado por Mojtaba Nabili et al. (31) se determinaron las sustituciones de los aminoácidos de la proteína Erg11p en 60 aislamientos de Candida glabrata resistentes a azoles. Se identificó que existía una sustitución en el aminoácido G236V en el sitio de unión de la enzima al fluconazol, lo que disminuía considerablemente la afinidad del medicamento por su diana biológica. Otras mutaciones H146Q y D234E localizadas cerca al sitio de unión de la misma enzima con los triazoles ejercen un efecto similar.

\section{Conclusiones}

La resistencia farmacológica desarrollada por Candida glabrata ha menoscabado la eficacia de las intervenciones con fluconazol y genera controversia ante su uso para tratar las candidiasis. La sobreexpresión de los genes CgCDR1, CgCDR2, CgSNQ2, PDH1, CgPdr1 y MDR1, cuyo resultado es el incremento de bombas de extrusión de fluconazol en el hongo, reduce la concentración de este en el interior de la célula fúngica. Ello se traduce en una menor efectividad farmacológica y, según estudios como efecto negativo colateral, incrementa la resistencia posterior a la exposición con el medicamento, puesto que se ha generado la necesidad de que la especie mute para defenderse del ataque farmacológico.

La modificación en la lanosterol 14-alfa-desmetilasa generada por mutaciones en el gen ERG11 impide que el fluconazol se una a esta y, por supuesto, su acción inhibitoria sobre este blanco molecular, lo que resta las posibilidades de que el fármaco actúe de manera eficaz en el control de las infecciones provocadas por Candida glabrata, en la medida en que no podrá bloquearse la síntesis de ergosterol por parte del microrganismo para la formación de su membrana.

Ante el panorama expuesto, es pertinente que en el momento de tomar decisiones frente a la intervención con fluconazol, un buen laboratorio establezca claramente la etiología de la enfermedad micótica, además de la identificación. También es necesario reconocer el perfil de resistencia de dicho hongo, puesto que constituye un elemento importante para acertar en la terapéutica y evitar de esta manera desperdiciar recursos y contribuir a la generación de más cepas resistentes.

\section{Referencias}

1. Falagas ME, Roussos N, Vardakas KZ. Relative frequency of albicans and the various non-albicans Candida spp. among candidemia isolates from inpatients in various parts of the world: a systematic review. Int J Infect Dis. 2010;14(11):e954-66. https://doi.org/10.1016/j.ijid.2010.04.006

2. Yeşilkaya A, Azap Ö, Aydın M, Akçil Ok M. Epidemiology, species distribution, clinical characteristics and mortality of candidaemia in a tertiary care university hospital in Turkey, 2007-2014. Mycoses. 2017;60(7):433-9. https:/ /doi.org/10.1111/myc.12618

3. Savastano C, de Oliveira Silva E, Gonçalves LL, Nery JM, Silva NC, Dias ALT. Candida glabrata among Candida spp. from environmental health practitioners of a Brazilian Hospital. Braz J Microbiol Publ Braz Soc Microbiol. 2016;47(2):367-72. https://doi.org/10.1016/j.bjm.2015.05.001

4. Goemaere B, Becker P, Van Wijngaerden E, Maertens J, Spriet I, Hendrickx M, et al. Increasing candidaemia incidence from 2004 to 2015 with a shift in epidemiology in patients preexposed to antifungals. Mycoses. 2018;61(2):127-33. https://doi.org/10.1111/myc.12714

5. Sasso M, Roger C, Sasso M, Poujol H, Barbar S, Lefrant J-Y, et al. Changes in the distribution of colonising and infecting Candida spp. isolates, antifungal drug consumption and susceptibility in a French intensive care unit: A 10-year study. Mycoses. 2017;60(12):770-80. https://doi.org/10.1111/myc.12661

6. de Bedout C, Gómez BL. Candida and candidiasis: the challenge continues for an early diagnosis. Infectio. 2010;14:s159-71. 
7. Valle GMM del. Candida glabrata: un patógeno emergente. Biociencias. 2016;10(1):89-102. https://doi.org/10.1 8041/2390-0512/bioc..1.2859

8. Rodríguez AZ, Gómez C de B, Restrepo CAA, Parra HH, Arteaga MA, Moreno AR, et al. [Susceptibility to fluconazole and voriconazole of Candida species isolated from intensive care units patients in Medellin, Colombia (2001-2007)]. Rev Iberoam Micol. 2010;27(3):125-9. https://doi.org/10.1016/j.riam.2010.04.001

9. Magalhães YC, Bomfim MRQ, Melônio LC, Ribeiro PCS, Cosme LM, Rhoden CR, et al. Clinical significance of the isolation of Candida species from hospitalized patients. Braz J Microbiol Publ Braz Soc Microbiol. 2015;46(1):117-23. https://doi.org/10.1590/S1517-838246120120296

10. Tapia P C. Candida glabrata. Rev Chil Infectol. 2008;25(4):293-293. http://dx.doi.org/10.4067/S0716-101820 08000400009

11. Olaechea P, Lerma FA, Martínez MP, Ordeñana JI, López-Pueyo MJ, Betolaza IS, et al. Evolución del consumo de antifúngicos en pacientes críticos. Estudio multicéntrico observacional, 2006-2010. Enferm Infecc Microbiol Clin. 2012;30(8):435-40. https://doi.org/10.1016/j.eimc.2012.02.006

12. Sanguinetti M, Posteraro B, Lass-Flörl C. Antifungal drug resistance among Candida species: mechanisms and clinical impact. Mycoses. 2015;58 Suppl 2:2-13. https://doi.org/10.1111/myc.12330

13. Florez J, Armijo JA, De Cost MA. Reacciones adversas a los medicamentos y farmacovigilancia. En: Flórez J, Armijo JA, editores. Farmacología humana. 5.. ed. Barcelona: Masson; 2008. p. 129-46.

14. Isaza G, Fuentes J, Marulanda T, Buriticá O, Machado J, Moncada J. Generalidades de farmacología. En: Isaza G, Fuentes J, Marulanda T, et al., editores. Fundamentos de farmacología en terapéutica. 6.. ed. Bogotá: Celsus; 2014. p. 27-31.

15. Moher D, Liberati A, Tetzlaff J, Altman DG, PRISMA Group. Preferred reporting items for systematic reviews and meta-analyses: the PRISMA statement. PLoS Med. 21 de julio de 2009;6(7):e1000097. https://doi.org/1 0.1371/journal.pmed.1000097

16. Deorukhkar SC, Saini S. Virulence factors attributed to pathogenicity of non albicans Candida species isolated from Human Immunodeficiency virus infected patients with oropharyngeal candidiasis. Ann Pathol Lab Med. 2015;2(2):A62-66. https://doi.org/10.1155/2014/456878

17. Ahmad KM, Kokošar J, Guo X, Gu Z, Ishchuk OP, Piškur J. Genome structure and dynamics of the yeast pathogen Candida glabrata. FEMS Yeast Res. 2014;14(4):529-35. https://doi.org/10.1111/1567-1364.12145

18. Muller H, Hennequin C, Gallaud J, Dujon B, Fairhead C. The asexual yeast Candida glabrata maintains distinct a and alpha haploid mating types. Eukaryot Cell. 2008;7(5):848-58. https://doi.org/10.1128/EC.00456-07

19. Bairwa G, Rasheed M, Taigwal R, Sahoo R, Kaur R. GPI (glycosylphosphatidylinositol)-linked aspartyl proteases regulate vacuole homoeostasis in Candida glabrata. Biochem J. 2014;458(2):323-34. https://doi.org/10.1042/ BJ20130757

20. Lesage G, Bussey H. Cell Wall Assembly in Saccharomyces cerevisiae. Microbiol Mol Biol Rev. 2006;70(2):317-43. https://doi.org/10.1128/MMBR.00038-05

21. Pontón J. La pared celular de los hongos y el mecanismo de acción de la anidulafungina. Rev Iberoam Micol. 2008;25(2):78-82. https://doi.org/10.1016/S1130-1406(08)70024-X

22. Chaffin WL, López-Ribot JL, Casanova M, Gozalbo D, Martínez JP. Cell wall and secreted proteins of Candida albicans: identification, function, and expression. Microbiol Mol Biol Rev MMBR. 1998;62(1):130-80

23. Okada H, Abe M, Asakawa-Minemura M, Hirata A, Qadota H, Morishita K, et al. Multiple functional domains of the yeast 1,3-beta-glucan synthase subunit Fks1 p revealed by quantitative phenotypic analysis of temperaturesensitive mutants. Genetics. 2010;184(4):1013-24. https://doi.org/10.1534/genetics.109.109892

24. Spanova M, Czabany T, Zellnig G, Leitner E, Hapala I, Daum G. Effect of lipid particle biogenesis on the subcellular distribution of squalene in the yeast Saccharomyces cerevisiae. J Biol Chem. 2010;285(9):6127-33. https://doi .org/10.1074/jbc.M109.074229

25. Base de datos en farmacología Drugbank. Fluconazole [Internet]. Washington: FDA. 2018 [citado 2018 oct 7]. Disponible en: https://www.drugbank.ca/drugs/DB00196 
26. Tuck SF, Patel H, Safi E, Robinson CH. Lanosterol 14 alpha-demethylase (P45014DM): effects of P45014DM inhibitors on sterol biosynthesis downstream of lanosterol. J Lipid Res. 1991;32(6):893-902.

27. Fonseca E, Silva S, Rodrigues CF, Alves CT, Azeredo J, Henriques M. Effects of fluconazole on Candida glabrata biofilms and its relationship with ABC transporter gene expression. Biofouling. 2014;30(4):447-57. https://d oi.org/10.1080/08927014.2014.886108

28. Castanheira M, Messer SA, Jones RN, Farrell DJ, Pfaller MA. Activity of echinocandins and triazoles against a contemporary (2012) worldwide collection of yeast and moulds collected from invasive infections. Int J Antimicrob Agents. 2014;44(4):320-6. https://doi.org/10.1016/j.jiantimicag.2014.06.007

29. Roetzer A, Gratz N, Kovarik P, Schüller C. Autophagy supports Candida glabrata survival during phagocytosis. Cell Microbiol. 2010;12(2):199-216. https://doi.org/10.1111/j.1462-5822.2009.01391

30. Chelsea M, Armstrong A, Armstrong E. Principios de quimioterapia. En: Brunton L, Chabner B, Knollman B, editores. Bases fisiopatológicas del tratamiento farmacológico. 4.. ed. Barcelona: Lippincott Williams and Wilkins; 2017. p. 661-74.

31. Nabili M, Abdollahi Gohar A, Badali H, Mohammadi R, Moazeni M. Amino acid substitutions in Erg1 1p of azoleresistant Candida glabrata: Possible effective substitutions and homology modelling. J Glob Antimicrob Resist. 2016;5:42-6. https://doi.org/10.1016/j.jgar.2016.03.003

32. Morace G, Perdoni F, Borghi E. Antifungal drug resistance in Candida species. J Glob Antimicrob Resist. 2014;2(4):254-9. https://doi.org/10.1016/j.jgar.2014.09.002

33. Morio F, Jensen RH, Le Pape P, Arendrup MC. Molecular basis of antifungal drug resistance in yeasts. Int J Antimicrob Agents. 2017;50(5):599-606. https://doi.org/10.1016/j.ijantimicag.2017.05.012

34. Flórez J, Peralta G, Mediavilla A. Enfermedades infecciosas. En: Flórez J, Armijo JA, editores. Farmacología humana. 5.. ed. Barcelona: Masson; 2008. p. 1301-16.

35. Sheppard D, Lampiris H. Fármacos quimioterapéuticos. En: Katzung BG, Masters SB, Trevor AJ, editores. Farmacología básica y clínica. 12.. ed. México: McGraw-Hill; 2013. p. 849-60.

36. Silva V, Díaz MC, Febré N. Vigilancia de la resistencia de levaduras a antifúngicos. Rev Chil Infectol. 2002;19 (suppl 2):149-56. http://dx.doi.org/10.4067/S0716-10182002019200016

37. Becher R, Wirsel SGR. Fungal cytochrome P450 sterol 14 $\alpha$-demethylase (CYP51) and azole resistance in plant and human pathogens. Appl Microbiol Biotechnol. 2012;95(4):825-40. https://doi.org/10.1007/s00253-012 $-4195-9$

38. Morace G, Perdoni F, Borghi E. Antifungal drug resistance in Candida species. J Glob Antimicrob Resist. 2014;2(4):254-9. https://doi.org/10.1016/j.jgar.2014.09.002

39. Biswas C, Chen SC-A, Halliday C, Kennedy K, Playford EG, Marriott DJ, et al. Identification of genetic markers of resistance to echinocandins, azoles and 5-fluorocytosine in Candida glabrata by next-generation sequencing: a feasibility study. Clin Microbiol Infect. 2017;23(9):676.e7-676.e10. https://doi.org/10.1016/j.cmi.2017.03. 014

40. Morio F, Jensen RH, Le Pape P, Arendrup MC. Molecular basis of antifungal drug resistance in yeasts. Int J Antimicrob Agents. 2017;50(5):599-606. https://doi.org/10.1016/j.ijantimicag.2017.05.012

41. Silva DB dos S, Rodrigues LMC, Almeida AA de, Oliveira KMP de, Grisolia AB. Novel point mutations in the ERG11 gene in clinical isolates of azole resistant Candida species. Mem Inst Oswaldo Cruz. 2016;111(3):192-9. https://doi.org/10.1590/0074-02760150400

42. Puri N, Manoharlal R, Sharma M, Sanglard D, Prasad R. Overcoming the heterologous bias: an in vivo functional analysis of multidrug efflux transporter, $\mathrm{CgCdr} 1 \mathrm{p}$ in matched pair clinical isolates of Candida glabrata. Biochem Biophys Res Commun. 2011;404(1):357-63. https://doi.org/10.1016/j.bbrc.2010.11.123

43. Clinical and Laboratory Standards Institute (CLSI). Methods for antifungal disk diffusion susceptibility testing of yeasts; approved guideline-Second Edition; 2009. CLSI Document M44-A2: [Internet]. 2009. Disponible en: https://clsi.org/media/1634/m44a2_sample.pdf 
44. Clinical and Laboratory Standards Institute (CLSI). Reference method for broth dilution antifungal susceptibility testing of yeasts; approved standard-Third Edition; 2008. CLSI Document M27-A3 [Internet]. 2008. Disponible en: https://clsi.org/media/1461/m27a3_sample.pdf

45. Clinical and Laboratory Standards Institute (CLSI). reference method for broth dilution antifungal susceptibility testing of yeasts; fourth informational supplement; 2012. CLSI Document M27-S4. [Internet]. 2012. Disponible en: https://clsi.org/media/1897/m27ed4_sample.pdf

46. Clinical and Laboratory Standards Institute (CLSI). Perfomance stands for antifungal susceptibility testing of yeasts; first edition; 2017. CLSI Document M60 [Internet]. 2017. Disponible en: https://clsi.org/standards/p roducts/microbiology/documents/m60/

47. Branco J, Ola M, Silva RM, Fonseca E, Gomes NC, Martins-Cruz C, et al. Impact of ERG3 mutations and expression of ergosterol genes controlled by UPC2 and NDT80 in Candida parapsilosis azole resistance. Clin Microbiol Infect. 2017;23(8):575.e1-575.e8. https://doi.org/10.1016/j.cmi.2017.02.002

48. Tapia C. Antifúngicos y resistencia. Rev Chil Infectol. 2012;29(3):357-357. http://dx.doi.org/10.4067/S0716-1 0182012000300020

49. Pfaller MA. Antifungal drug resistance: mechanisms, epidemiology, and consequences for treatment. Am J Med. 2012;125(1 Suppl):S3-13. https://doi.org/10.1016/j.amjmed.2011.11.001

50. Abbes S, Amouri I, Sellami H, Neji S, Trabelsi H, Cheikhrouhou F, et al. Changes in genotype and fluconazole susceptibility of isolates from patients with Candida glabrata in Tunisia. Thérapie. 2014;69(5):449-55. https:// doi.org/10.2515/therapie/2014059

51. López-Ávila K, Dzul-Rosado KR, Lugo-Caballero C, Arias-León JJ, Zavala-Castro JE. Mecanismos de resistencia antifúngica de los azoles en Candida albicans: una revisión. Rev Bioméd. 2016;27(3):127-36. http://dx.doi.or g/10.32776/revbiomed.v27i3.541

52. Vermitsky J-P, Edlind TD. Azole Resistance in Candida glabrata: coordinate upregulation of multidrug transporters and evidence for a Pdr1-Like transcription factor. Antimicrob Agents Chemother. 2004;48(10):3773-81. https ://doi.org/10.1128/AAC.48.10.3773-3781.2004

53. Kanafani ZA, Perfect JR. Resistance to antifungal agents: mechanisms and clinical impact. Clin Infect Dis. 2008;46(1):120-8. https://doi.org/10.1086/524071

54. Pfaller MA, Castanheira M, Lockhart SR, Ahlquist AM, Messer SA, Jones RN. Frequency of decreased susceptibility and resistance to echinocandins among fluconazole-resistant bloodstream isolates of Candida glabrata. J Clin Microbiol. 2012;50(4):1199-203. https://doi.org/10.1128/JCM.06112-11

55. Ghannoum MA, Rice LB. Antifungal agents: mode of action, mechanisms of resistance, and correlation of these mechanisms with bacterial resistance. Clin Microbiol Rev. 1999;12(4):501-17

56. Balouiri M, Sadiki M, Ibnsouda SK. Methods for in vitro evaluating antimicrobial activity: A review.J Pharm Anal. 2016;6(2):71-9. doi 10.1016/j.jpha.2015.11.005

57. Sakagami T, Kawano T, Yamashita K, Yamada E, Fujino N, Kaeriyama M, et al. Antifungal susceptibility trend and analysis of resistance mechanism for Candida species isolated from bloodstream at a Japanese university hospital. J Infect Chemother. 2019;25(1):34-40. https://doi.org/10.1016/j.jiac.2018.10.007

58. Rocha DAS, Sa LFR de, Pinto ACC, Junqueira M de L, Silva EM da, Borges RM, et al. Characterisation of an ABC transporter of a resistant Candida glabrata clinical isolate. Mem Inst Oswaldo Cruz. 2018;113(4):e170484. ht tps://doi.org/10.1590/0074-02760170484

59. Szweda P, Gucwa K, Romanowska E, Dzierz Anowska-Fangrat K, Naumiuk Ł, Brillowska-Da Browska A, et al. Mechanisms of azole resistance among clinical isolates of Candida glabrata in Poland. J Med Microbiol. 2015;64(6):610-9. https://doi.org/10.1099/jmm.0.000062

60. Holmes AR, Keniya MV, Ivnitski-Steele I, Monk BC, Lamping E, Sklar LA, et al. The monoamine oxidase A inhibitor clorgyline is a broad-spectrum inhibitor of fungal ABC and MFS transporter efflux pump activities which reverses the azole resistance of Candida albicans and Candida glabrata clinical isolates. Antimicrob Agents Chemother. 2012;56(3):1508-15. https://doi.org/10.1128/AAC.05706-11 
61. Salazar SB, Wang C, Münsterkötter M, Okamoto M, Takahashi-Nakaguchi A, Chibana H, et al. Comparative genomic and transcriptomic analyses unveil novel features of azole resistance and adaptation to the human host in Candida glabrata. FEMS Yeast Res. 2018;18(1). https://doi.org/10.1093/femsyr/fox079

62. Tsai H-F, Sammons LR, Zhang X, Suffis SD, Su Q, Myers TG, et al. Microarray and molecular analyses of the azole resistance mechanism in Candida glabrata oropharyngeal isolates. Antimicrob Agents Chemother. 2010;54(8):3308-17. https://doi.org/10.1128/AAC.00535-10

63. Hou X, Xiao M, Wang H, Yu S-Y, Zhang G, Zhao Y, et al. Profiling of PDR1 and MSH2 in Candida glabrata bloodstream isolates from a multicenter study in China. Antimicrob Agents Chemother. 2018;62(6). https:// doi.org/10.1128/AAC.00153-18

\section{Notas}

* Artículo de revisión

Conflictos de interés: los autores declaran no tener conflictos de interés.

\section{Licencia Creative Commons CC BY 4.0}

Cómo citar este artículo: Cárdenas Parra LY, Pérez Cárdenas JE. Mecanismos de resistencia a fluconazol expresados por Candida glabrata: una situación para considerar en la terapéutica. Investig Enferm Imagen Desarr. 2020;22. https://doi.org/10.11144/Javeriana.ie22.mrfe

Financiación: ninguna 